\title{
Ambientes restauradores: Definição, histórico, abordagens e pesquisas
}

\author{
Sandra Christina Gressler \\ Isolda de Araújo Günther \\ Universidade de Brasilia
}

\begin{abstract}
Resumo
Objetiva-se identificar referenciais teóricos que elucidem o conceito de ambientes restauradores (restorative environment). Examinam-se sua definição, contextualização histórica, as diferentes abordagens teóricas, os avanços nas pesquisas. Foram analisados artigos publicados entre os anos de 1991 e 2011 em periódicos científicos disponíveis no portal da CAPES. Verifica-se a atualidade do tema pelo aumento de estudos publicados nos últimos três anos. Pesquisas sobre ambientes restauradores investigam os fatores que auxiliam na promoção do bem-estar do ser humano, e reforçam o movimento interdisciplinar entre a psicologia e, por exemplo, a saúde e prevenção, a educação, a arquitetura e o planejamento urbano.
\end{abstract}

Palavras-chave: ambientes restauradores; psicologia ambiental; teoria da recuperação psicofisiológica ao estresse; teoria da restauração da atenção.

\begin{abstract}
Restorative environments: Definition, history, approaches and research. This article aims to identify theoretical references, which elucidate the concept of restorative environments; examines its definition, historical background, different theoretical approaches and advances in research. Articles published between 1991 and 2011 in scientific journals available on the website of CAPES were analyzed. The current interest in the field was verified through an increase of studies published over the last three years. Studies on restorative environments seek to investigate the factors that help to promote the well-being of people and reinforce the interdisciplinary movement between psychology and, for example, health and prevention, education, architecture and urban planning.
\end{abstract}

Keywords: restorative environments; environmental psychology; psychophysiological stress recovery theory; attention restoration theory.

\section{Resumen}

Ambientes restauradores: Definición, descripción, enfoque e investigación. Se tiene como objetivo identificar los referenciales teóricos que aclaran el concepto de ambientes restauradores (restorative environment). Se examinan su definición, contextualización histórica, los diversos enfoques teóricos, los avances en la investigación. Se analizaron los artículos publicados entre los años de 1991 y 2011 en revistas científicas disponibles en el sitio de CAPES. Se verifica la actualidad del tema por el aumento de los estudios publicados en los últimos tres años. Investigaciones sobre ambientes restauradores estudian los factores que asisten a la promoción del bienestar del humano, y refuerzan el movimiento interdisciplinar entre la psicología y, por ejemplo, la salud y la prevención, la educación, la arquitectura y el planeamiento urbano.

Palabras clave: ambientes restauradores; psicología ambiental; teoría de la recuperación psicofisiológica al estrés; teoría de la restauración de la atención.

$\mathrm{A}$ s demandas do convívio social, as atividades diárias e as exigências profissionais requerem uma atenção constante que, agregadas à falta de condições ideais, como o barulho excessivo, a aglomeração, o trânsito, e a violência, causam estresse muitas vezes crônico.

Nesse contexto, duas frentes de pesquisa buscam detectar os fatores que afetam o bem estar do ser humano. A primeira realiza estudos sobre os fatores ambientais causadores de estresse. A segunda busca identificar ambientes restauradores (restorative environments), capazes de restabelecer os indivíduos afetados pelo processo psicofisiológico de estresse.

\section{Objetivo e critérios de elegibilidade}

O presente trabalho tem por objetivo apresentar o conceito 
ambientes restauradores, sua definição, contextualização histórica, as diferentes abordagens e os avanços nas pesquisas. Para tanto, foram analisadas as publicações em periódicos científicos entre os anos de 1991 e 2011, obtidos do portal da CAPES (http://www.periodicos.capes.gov.br/), durante o mês de janeiro de 2012.

Como o termo restorative environment não apresenta uma tradução sedimentada no meio científico brasileiro, optou-se por utilizar diversos descritores em português visando encontrar um maior número de pesquisas a respeito do assunto. Para a busca às pesquisas publicadas na língua portuguesa foram utilizadas, dentro do portal da CAPES, as bases de dados SciELO e PePSIC com os descritores: ambiente restaurador, jardins terapêuticos, recuperação psicofisiológica ao estresse e teoria da restauração da atenção (attention restoration theory - ART).

Com o uso do descritor restorative environment, a busca foi realizada no portal da CAPES dentro as bases de dados Science Direct, Scopus, Webof Science, PsycoINFO, APA PsycNET, EBSCO, Mary Ann Liedert e Sage. Quando apresentado como opção na base de dados, foi utilizado o recurso de filtros "abstract, title, keywords". Nas demais bases de dados prevaleceram, na busca, "todos os índices" sendo que o termo, por ser composto, teve aspas em sua formatação. Como critério para a escolha das bases de dados levou-se em consideração a sugestão oferecida durante treinamento realizado na biblioteca da universidade (nome retirado para evitar a identificação de autoria do manuscrito).

Uma vez identificados, os artigos a serem revisados foram sistematicamente analisados, procurando-se classificá-los quanto aos seguintes aspectos: 1) autores; 2) ano da publicação; 3 ) título da publicação; 4) nome do periódico científico (volume e/ou número); 5) finalidade do estudo (gerar conhecimento, instrumento ou tecnologia); 6) tipo de instrumento (questionário, escala, entrevista, observação, análise de documento, testes/ prova, fotos, filme, outros); e 7) relação com o tema restorative environment (direta, indireta).

Entre as pesquisas publicadas nas bases de dados SciELO e PePSIC com os descritores ambiente restaurador, jardins terapêuticos, recuperação psicofisiológica ao estresse e teoria da restauração da atenção foram localizados cinco artigos na base de dados SciELO utilizando método Google acadêmico, e trinta artigos pela opção proximidade léxica, que aponta as relações entre os termos por meio da comparação de sua cadeia de caracteres. No caso em pauta, a similaridade léxica foi inferior a 0,5 , indicativa de uma correspondência fraca que se situa entre 0,55 e 0,59 . Entre as pesquisas encontradas duas, relacionadas à área de saúde, abordavam o tema ambientes restauradores.

Na busca realizada com o descritor restorative environment, foram encontrados pouco mais de 300 artigos. Dessa busca foram excluídos capítulos de livros, dada a limitada capacidade de acessá-los, e artigos repetidos, sendo considerados os artigos publicados em inglês e excluídos os artigos em alemão e finlandês. Entre esses estudos, 239 foram selecionados por se tratarem de artigos relacionados à abordagem desejada para essa investigação. Entre os artigos selecionados, foi possível ter acesso a 146 textos completos de artigos que mencionaram e pesquisaram de forma direta os temas ambientes e/ou atividades restauradoras.

Fazem parte, portanto, desta revisão os 146 artigos selecionados que mantinham relação direta com o tema, além de artigos e capítulos de livros relacionados ao tema, mas que foram encontrados de maneira não sistemática. Entre os capítulos de livros destacamos dois publicados no Brasil: Alves (2011) e Alves e Gulwadi (2008). Sublinha-se o acréscimo das publicações sobre o tema, uma vez que, cerca de cinquenta por cento das pesquisas encontradas na presente busca, foram divulgadas entre os anos de 2008 e 2011.

\section{Definição e contextualização histórica}

As pesquisas sobre ambientes restauradores (restorative environments) ganharam maior visibilidade na década de 1980 , quando alguns pesquisadores (Altman \& Wohlwill, 1983; S. Kaplan \& Kaplan, 1982; K. Korpela, 1989) conduziram estudos de atributos ambientais. Nesse período, algumas áreas específicas da psicologia ligadas, por exemplo, ao estudo do estresse e da avaliação ambiental receberam uma maior atenção (Holahan, 1986). Buscava-se compreender os fatores que diferenciavam as sensações de prazer ou desprazer experimentadas em determinado ambiente. Concomitantemente, estudos sobre apego ou desapego ao lugar, identidade e significado do lugar, ambientes calmos ou estressantes foram alguns dos temas em voga.

O termo ambientes restauradores foi sugerido a partir das teorias propostas por Rachel e Stephen Kaplan e Roger Ulrich (R. Kaplan \& Kaplan, 1989; S. Kaplan, 1995; Ulrich, 1983, 1984). Nesses estudos, estresse foi definido como o processo pelo qual um indivíduo responde psicofisiologicamente, por meio de comportamentos, a uma situação que desafia ou ameaça seu bem estar (Baum, Fleming, \& Singer, 1985), percebida em autorrelatos de emoções negativas. Opondo-se à noção de estresse, surge um construto denominado restoration, definido como o processo de restauração, recuperação ou restabelecimento dos aspectos físicos, psicológicos ou da capacidade social, perdidos pelo esforço contínuo. Alerta-se que as pesquisas a respeito de fatores estressantes não necessariamente explicam o fator restaurador (Hartig \& Staats, 2003). Mesmo assim, ainda é possível encontrar elementos estressantes comuns e, de acordo com Evans e Cohen (1987), também é possível encontrar elementos restauradores comuns.

O termo restoration abrange muitos processos, sendo enfatizados por alguns pesquisadores os aspectos psicofisiológicos (Evans \& Cohen, 1987). Apesar de a redução do estresse, proposta por Ulrich (Ulrich, 1983, 1984; Ulrich et al., 1991), e a restauração da capacidade de atenção direta, proposta por Rachel e Stephen Kaplan (1989), serem dois processos distintos, pondera-se que eles podem ocorrer simultaneamente. Dessa forma, acontece uma possível unidade entres as teorias com relação à expressão ambientes restauradores. Percebe-se, no entanto, que a teoria formulada por (R. Kaplan \& Kaplan, 1989) apresenta uma abordagem teórica estruturada, sendo possível reconhecer os estudos nela apoiados. Em contrapartida, a teoria proposta por Ulrich (1983) é utilizada em grande parte por estudos com abordagens relacionadas ao bem estar humano ou à relação positiva com os ambientes naturais e guarda semelhança 
com pesquisas relacionadas aos temas landscape therapy, horticulture therapy, therapeutic gardening ou, healing garden.

Os ambientes com características que promovem restauração são cada vez mais importantes para o ser humano moderno (Hartig \& Staats, 2003), vistas as exigências impostas às pessoas, pelo cotidiano.

\section{Ambientes restauradores: Teorias}

Duas linhas de pesquisa, conduzidas separadamente, tiveram uma contribuição direta para a elaboração do conceito de ambientes restauradores. Uma delas, proposta por Ulrich (Ulrich, 1983, 1984; Ulrich et al., 1991), está ligada à redução do estresse. Outro conjunto de estudos foi proposto por Rachel e Stephen Kaplan (R. Kaplan, 1983, 1984; R. Kaplan \& Kaplan, 1989; S. Kaplan, 1987, 1995; S. Kaplan \& Kaplan, 1982; S. Kaplan \& Talbot, 1983) e está ligado à restauração da capacidade de atenção.

\section{Teoria da recuperação psicofisiológica ao estresse}

Roger Ulrich (1983) propôs uma estrutura de redução psicológica do estresse. Essa estrutura embasa sua teoria, conhecida como recuperação psicofisiológica ao estresse (psychophysiological stress recovery). Em sua teoria psicoevolucionista, Ulrich (1983) basicamente enfatizou a percepção visual e estética de certos ambientes à resposta afetiva associada. O autor postulou que, por uma questão de sobrevivência, o ser humano se utiliza de estratégias comportamentais, que requerem decisões afetivas, como aproximação ou repulsão, além de uma mobilização simultânea de recursos fisiológicos para atender a tais estratégias. A exigência ligada ao excesso de tomada de decisões pode causar estresse. As consequências do estresse são autorrelatos de emoções negativas e, em um curto período de tempo, mudanças negativas do sistema fisiológico e aumento da atenção automática, isto é, da vigilância.

Segundo a abordagem de Ulrich, estar cercado por fatores que estimulam a aproximação e desencorajam certos comportamentos é fundamental para o bem estar e a sobrevivência humana. As experiências de ambientes físicos, visualmente prazerosos, podem auxiliar na redução do estresse, uma vez que desencadeiam emoções positivas, mantêm o estado de atenção não vigilante, diminuem os pensamentos negativos e possibilitam o retorno à excitação fisiológica (physiological arousal) para níveis mais moderados. Tudo isso parece levar à suposição de que as respostas restauradoras são imediatas, a partir da visão de parâmetros positivos em determinado ambiente (van Den Berg \& Custers, 2011).

Uma vez que as propriedades visuais influenciam preferências estéticas e despertam o interesse, elas podem ter moderada profundidade, moderada complexidade e presença de um ponto focal. Ulrich (1983) considerou alguns aspectos da natureza como capazes de promover recuperação psicofisiológica ao estresse, como a água e a vegetação, principalmente gramados e árvores.

Um dos estudos iniciais de Ulrich (1984) foi realizado em um hospital na Pensilvânia, entre 1972 e 1981. Os resultados indicaram que pacientes que haviam sido submetidos a cirurgia e que apresentavam o mesmo quadro clínico, quando colocados em leitos hospitalares que possibilitavam a visão da natureza através da janela do hospital, tiveram em geral menor tempo de internação pós-operatório, receberam menos comentários negativos na avaliação das enfermeiras e necessitaram de menor quantidade de analgésicos. $\mathrm{O}$ mesmo não aconteceu com pacientes que se encontravam em quartos com janelas com vista para uma parede de tijolos de um prédio vizinho. Os resultados dessa pesquisa sugerem que apenas um vislumbre da natureza pode possibilitar a recuperação do estresse.

\section{Teoria da restauração da atenção}

Stephen e Rachel Kaplan (R. Kaplan \& Kaplan, 1989; S. Kaplan, 1995), após anos de pesquisa em cognição ambiental e preferências ambientais, desenvolveram a teoria da restauração da atenção (attention restoration theory- ART). Os Kaplan postularam que, após horas de concentração da atenção, ou mesmo de exposição ao estresse da vida cotidiana, poder-se ia experimentar fadiga no processo de atenção, com necessidade para o cérebro humano de um momento para descansar e retomar a atenção.

Wells (2000) e Ouellette, Kaplan e Kaplan (2005) comentaram que o estudo da restauração da atenção foi influenciado por William James em sua publicação de 1892. Segundo Wells (2000), James enfatizou o empenho no conceito de atenção voluntária. No entanto, não incluiu em seu estudo a possibilidade da atenção voluntária ser susceptível à fadiga. Outra contribuição importante diz respeito ao estudo de S. Kaplan (1987) sobre os componentes cognitivos na avaliação ambiental, sugerindo que muitas decisões humanas são tomadas de modo não consciente. Para o autor, a qualidade da interrelação de um sujeito com o ambiente está relacionada às ações inerentes ao indivíduo e aos parâmetros de informação oferecidos pelo ambiente.

S. Kaplan (1995) argumentou que os indivíduos necessitam de um esforço constante para não perderem o foco de sua atenção direta para algo mais interessante. Essa luta diária para manter a concentração, acaba desencadeando um processo de fadiga. Os efeitos negativos da fadiga provocada pela atenção direta são: a irritabilidade, a falta de habilidade para planejar, a sensibilidade reduzida para perceber sinais ligados às relações interpessoais, o controle pessoal reduzido e o aumento de erros em atividades que exigem atenção direta. $\mathrm{O}$ conceito da fadiga de atenção direta proposto por Kaplan tem muito em comum com a ideia dos efeitos colaterais de estresse ou sobrecarga de informações para o cérebro. S. Kaplan (1995) enfatizou que, só no mundo moderno, a divisão entre o importante e o interessante tornou-se extrema, porque anteriormente, o que era importante para a evolução humana era fascinante por sua própria natureza e, portanto, não necessitava de atenção dirigida.

Segundo a teoria da restauração da atenção (ART), a experiência de restauração da atenção pressupõe alguns processos. A princípio, S. Kaplan (1995) sugeriu, como fatores promotores de restauração, os processos: fascination, being away, extent, e compatibility, aqui traduzidos por fascinação, afastamento, extensão e compatibilidade. Outras pesquisas (Herzog, Black, Fountaine, \& Knotts, 1997; Herzog, Maguire, \& Nebel, 2003; Karmanov \& Hamel, 2008; Perrin \& Benassi, 
2009; van den Berg, Koole, \& van der Wulp, 2003) detalharam a teoria e os processos envolvidos, apontando tanto as experiências restauradoras quanto os ambientes restauradores (S. Kaplan \& Talbot, 1983).

Sob essa ótica, fascinação é a atenção involuntária, que não exige esforço ou inibição de estímulos concorrentes, que permite ao sistema de atenção fatigado descansar e restaurar a capacidade de atenção dirigida. A fascinação pode ocorrer em uma diversidade de cenários e situações interessantes, não demanda esforço e pode variar em intensidade com dimensões soft ou hard (S. Kaplan, 1995).

A fascinação soft é caracterizada por uma intensidade moderada e, geralmente, centrada em estímulos esteticamente agradáveis, que permitem a oportunidade de reflexão, promovendo de maneira mais eficiente a restauração da atenção (Felsten, 2009). A beleza estética, componente da fascinação soft, deve gerar uma correlação positiva entre preferência e restauração. Esta última, caracterizada por níveis moderados da atenção "sem esforço" que se agregam aos aspectos relacionados à beleza estética do cenário (R. Kaplan, Kaplan, \& Ryan, 1998; S. Kaplan, 1995). Herzog et al. (1997), mostraram evidências que suportam a distinção entre fascinação hard e soft, bem como entre os diferentes benefícios restauradores associados à fascinação soft. Fascinação soft é comum em ambientes naturais, como, por exemplo, ver um pôr do sol, ou o suave balaço dos galhos de árvores ao vento. A combinação de fascinação e extensão, juntamente com aspectos estéticos, captura efetivamente o conceito de fascinação soft no propósito de reflexão (Ouellette et al., 2005).

A distinção em relação à fascinação hard é que essa aguça a atenção e geralmente não permite reflexão. Fascinação hard, por exemplo, pode ocorrer ao se visualizar um evento esportivo muito competitivo. Essa forma de atenção não permite alcançar os benefícios mais profundos de uma experiência reparadora, como a reflexão sobre questões importantes.

Rachel Kaplan e Stephen Kaplan (1989) conceberam que o primeiro fator, fascinação, sozinho, não causa restauração da atenção, sendo necessário um segundo fator, o afastamento.

Afastamento envolve possibilidades geográficas e/ou psicológicas de estar afastado do contexto usual, das experiências da vida quotidiana, da necessidade de atenção direta, focalizada. Mas, o que parece ser necessário para um ambiente ser restaurador é ser capaz de proporcionar uma distância mais conceitual do que física, já que um ambiente novo ou a novidade, por si só, não é restaurador, mas se torna restaurador caso promova uma mudança nos nossos pensamentos, relacionada às pressões e obrigações da vida cotidiana. Na definição do ART, afastamento apresenta um componente físico (na definição diferente do habitual), e um componente psicológico (ser capaz de escapar das distrações indesejadas e das lembranças de suas obrigações diárias). Segundo Laumann, Gärling, e Stormark (2001) o afastamento se aproxima mais do componente de fuga, de escapada, do que do componente de novidade.

Um terceiro fator, extensão, torna necessária a imersão em um ambiente físico coerente ou em um ambiente conceitual suficientemente planejado para possibilitar exploração e interpretação, ou seja, um ambiente que possua alcance suficiente para manter a interação sem provocar tédio, durante um período de tempo. Extensão refere-se às propriedades de conectividade e alcance em um determinado ambiente. Em primeiro lugar, um ambiente restaurador é percebido como um todo em que todos os elementos estão relacionados de forma coerente. Em segundo lugar, é abrangente o suficiente para engajar a mente, porque promete muito mais para explorar do que aquilo que é imediatamente percebido, "não se refere necessariamente à extensão física, mas envolve o senso de pertença... assim como o conhecimento de que esse ambiente é rico e dá margem à futura exploração" (Alves, 2011).

O quarto e último fator de restauração da atenção corresponde à compatibilidade e refere-se ao encontro entre as inclinações pessoais, os propósitos e o suporte do ambiente para determinadas atividades e as possíveis ações no ambiente. É a união entre inclinações e propósitos pessoais que pode vir a evitar o esforço mental exaustivo. Esse fator guarda semelhança com o conceito de affordance, isto é, a relação recíproca entre ambiente e ser vivo, observando a complementaridade (Günther, 2011).

O terceiro e quarto fatores, extensão e compatibilidade, fazem referência às características do lugar que possam promover restauração. Este espaço deve ser suficiente para possibilitar exploração e ser coerente o suficiente para fazer sentido, além de dar apoio à atividade proposta pelo indivíduo, que deve ter inclinação e capacidade para tais atividades. Resulta em um encontro das necessidades e capacidades do indivíduo com relação ao que o ambiente proporciona e possibilita. Para Rachel e Stephen Kaplan (1989) quanto maior o encontro dessas condições, maior será o fator restaurador.

Os quatro fatores propostos na ART associam-se às propriedades do ambiente nos quais os seres humanos desencadeiam processos mentais ou estados que contribuem para as experiências restauradoras. Alguns aspectos podem surgir, como: mistério, coerência, ambientes preferidos, o afastamento para realizar algo diferente em um local novo e o afastamento como uma forma de escape. Laumann et al. (2001) distinguiram o papel dos quatro componentes e, para os autores, o fator afastamento implica em relaxamento. Por sua vez, Herzog et al. (2003) forneceram dados empíricos ao mostrar que os quatro componentes têm eficácias relativamente diferentes como preditores do potencial de restauração. Os autores sugerem que compatibilidade e afastamento são mais poderosos que extensão e fascinação.

As características restauradoras concernentes às avaliações de preferência por diferentes ambientes também foram examinadas. Em um dos estudos, Laumann et al. (2001) verificaram que o fascínio, a novidade, a fuga, a extensão e a compatibilidade poderiam prever as preferências por determinado ambiente. Os autores sugerem, por meio de vídeos de passeios na floresta, no parque, na praia, na cidade e em montanhas com neve, que as avaliações das características restauradoras foram capazes de predizer a preferência por cinco ambientes diferentes, sendo a compatibilidade o fator preditivo mais importante para todos os ambientes.

Utilizando-se como base o conceito de ambientes restauradores, foram desenvolvidas diferentes escalas que avaliam a fascinação, o afastamento, a extensão e a 
compatibilidade, propostos na teoria ART. Utilizada para medir as propriedades percebidas no ambiente quanto à capacidade de restauração, foi desenvolvida a escala de restauratividade percebida (perceived restorativeness scale - PRS) (Hartig, Korpela, Evans, \& Garling, 1997), sendo elaborada no mesmo ano a escala revisada da restauração percebida (further development of a measure of perceived environmental restorativeness) (Hartig, Kaiser, \& Bowler, 1997). Laumann et al. (2001) propuseram como alternativa a escala de componentes restauradores (restorative components scale - RCS), defendendo que os quatro itens avaliados são construtos factíveis de serem analisados separadamente, uma vez que o afastamento está muito próximo à ideia de escapar. A escala de autorrestauração (self-rating restoration scale - RS) proposta por Han (2003) visa medir as qualidades restauradoras do ambiente, levando-se em consideração as questões emocionais, fisiológicas, cognitivas e comportamentais. A escala para medir os componentes restauradores percebidos por crianças (perceived restorative components scale for children- PRCS-C), desenvolvida por Bagot (2004), após análise fatorial dos itens, indicou um modelo com os fatores correspondendo à proposta de ART.

\section{Complementaridade entre as teorias}

Levando-se em consideração as duas linhas de pesquisa que estudam ambientes restauradores, é possível sugerir uma complementaridade entre as teorias propostas por Rachel e Stephen Kaplan (1989) e Ulrich (1983).

A elevada atenção psicológica e os efeitos negativos característicos do estresse podem ocorrer mesmo sem a fadiga do sistema de atenção concentrada, mesmo que a fadiga possa ser característica dos efeitos pós-estresse (Hartig, Evans, Jamner, Davis, \& Gärling, 2003). As teorias de restauração da atenção e da redução do estr esse diferem, na ênfase que dão aos aspectos emocionais, fisiológicos e aos fatores de atenção, bem como em suas especificações com relação às condições antecedentes ao processo restaurador.

Sinteticamente, parece adequado afirmar que a estrutura de redução do estresse, proposta por Ulrich (Ulrich, 1983; Ulrich et al., 1991), assume uma condição antecedente de estresse psicofisiológico, definido como o processo de resposta emocional, psicológica e comportamental, quando o bem-estar é desafiado ou ameaçado.

Outra diferenciação entre a restauração da atenção e a redução do estresse diz respeito à duração do processo de restauração e à questão da persistência dos efeitos serem imediatos, ou não. A abordagem das questões de redução do estresse psicofisiológico valoriza o momento em que a pessoa se encontra em local esteticamente prazeroso. Efeitos a longo prazo são possíveis, mas foram pouco discutidos. Em contraste, a teoria da restauração da atenção (ART) permite uma longa duração em uma experiência de restauração na qual o indivíduo pode passar por sucessivos estágios ou níveis. O alcance de níveis sucessivos requer aumento de tempo e intensidade dos fatores envolvidos na experiência restauradora (K. Korpela \& Hartig, 1996).

Face ao exposto, pondera-se que as duas teorias podem ser verificadas simultaneamente e, também, sob alguns aspectos, de forma independente uma da outra, com diferentes resultados, emergindo em diferentes períodos de tempo. As duas teorias também guardam semelhança quando atribuem funções restauradoras aos ambientes naturais (Hartig et al., 2003).

\section{Ambientes restauradores: Estudos empíricos}

Os estudos a respeito de experiências e/ou ambientes restauradores se iniciaram com as pesquisas relacionadas à preferência ambiental. Dessa forma, na busca de um modelo experimental, alguns estudos relacionados ao tema utilizaram um método que intercala momentos de estresse (testes de concentração, provas, filmes, etc.) com momentos de restauração (fotos de paisagens, filmes de ambientes naturais, passeios em parques) com o objetivo de ter uma pré e uma pós-medição, utilizando relatos obtidos por meio de questionários e entrevistas ou obtidos a partir da comparação dos índices encontrados nos diferentes momentos de estresse ou de restauração, em testes de memória, medição da pressão arterial, tensão muscular, pulsação, condutividade da pele, níveis de cortisol na saliva.

Uma vez que muitas pesquisas utilizam instrumentos que fazem a mediação, como, por exemplo, fotos ou filmes sobre a natureza, alguns pesquisadores começaram a indagar se não seria possível conseguir a restauração da atenção por meio de elementos mediadores. Iniciaram, então, questionamentos a respeito das possíveis diferenças entre ambientes restauradores originais, ao vivo, e ambientes restauradores mediados (de Kort \& Ijsselsteijn, 2006). Análises a respeito da mediação indicam que os efeitos positivos da exposição à natureza são parcialmente mediados pelo aumento do contato com ela, mas não são mediados por elevar a capacidade da atenção (Mayer, Frantz, Bruehlman-Senecal, \& Dolliver, 2009).

Em um estudo com modelo experimental, utilizando uma sala com uma TV de plasma que projetava cenas da natureza, uma sala com uma janela para um parque e uma sala sem janelas ou aparelho de televisão, concluiu-se que a janela de vidro com vista para um parque era significativamente mais restauradora do que a parede em branco (Kahn Jr et al., 2008). Comparandose com outros estudos, foi possível afirmar que imagens vistas em uma TV de plasma parecem ser uma opção melhor do que nenhuma natureza. No entanto, não é tão eficiente para restauração da atenção como visualizar a verdadeira natureza, o que levou Kahn e seus coautores a concluírem que os seres humanos adaptam-se à perda de natureza real, mas, ao fazê-lo, sofrem custos psicológicos.

Um estudo (Mayer et al., 2009) analisou diferentes grupos, convidados a refletirem sobre um problema diário relativamente menor em suas vidas. Esses grupos foram distribuídos em quatro ambientes distintos. Os autores concluíram que a resolução de problemas pessoais foi superior para os participantes expostos à natureza real, comparados aos participantes expostos à natureza virtual.

Kuo e Sullivan (2001) investigaram se as pessoas privadas das qualidades restauradoras possíveis no contato com a natureza reagem mais agressivamente. Agressividade tem sido associada à impulsividade, sendo sugerida uma relação entre a exposição à natureza e o autocontrole (Taylor, Kuo, \& Sullivan, 2002). As conclusões sobre a agressão e a autodisciplina também foram relatadas no ambiente do trânsito. Um estudo comparou as 
respostas fisiológicas de indivíduos que assistiram a um vídeo de condução que simulava um veículo percorrendo um trajeto com natureza, com indivíduos que assistiram a um vídeo de condução de um veículo que simulava o trajeto percorrido em ambientes construídos (Parsons, Tassinary, Ulrich, Hebl, \& Grossman-Alexander, 1998). Não só o grupo que simulava dirigir em estradas que percorriam ambientes naturais apresentou níveis mais baixos de estresse, como também se recuperou mais rapidamente do estresse induzido por esse experimento.

Ao se pesquisar sobre as propriedades restauradoras de atividades como caminhadas e/ou jardinagem, ou mesmo, propriedade restauradoras das plantas no ambiente construído (Bringslimark, Hartig, \& Patil, 2009) argumentou-se que os resultados poderiam estar ligados aos benefícios oriundos da exposição à luz solar. Pesquisas a respeito dos benefícios fisiológicos positivos à exposição à luz solar são facilmente comprovadas de forma empírica (van den Berg, 2005).

Até o momento, os resultados das pesquisas não permitem afirmar se as pessoas preferem locais naturais quando estão com humor positivo ou quando estão com o humor em baixa. Para Regan e Horn (2005), até o ano de 2005, não existia na literatura o registro de pesquisas sobre o desejo aparente dos indivíduos de estarem próximos à natureza quando moderadamente estressados, ou mesmo devido a fatores sociodemográficos, como o ambiente onde vivem, a idade, ou outros fatores, como o tipo de formação e emprego, ou a preferência por determinado hobbie.

Algumas críticas aos procedimentos são levantadas. Bourassa (1990) comenta que um número de pesquisas aponta para o fato de os seres humanos preferirem ambientes naturais a ambientes urbanos. Para o autor, os ambientes são experimentados, primeiramente, de modo cultural, isto é, envolvendo as relações sociais. No entanto, os pesquisadores em seus estudos sobre preferência, provavelmente, não capturam o significado para nenhum grupo cultural específico, nem mesmo para os indivíduos que fazem parte do estudo.

Dessa forma, estudos mais recentes sobre ambientes restauradores não questionam apenas o fator de restauração oferecido por ambientes natural versus artificial, mas passam por novas esferas de pesquisas envolvendo: (a) contexto social como ambiente restauradores; (b) ambientes restauradores para diferentes faixas etárias e/ou ao longo do curso de vida; (c) características individuais e/ou grupais, influenciando determinado ambiente restaurador, entre outras (Hartig \& Staats, 2003).

Acredita-se que o significado de experiências restauradoras provém da inter-relação dos componentes físicos e sociais, e pode ser caracterizado por diferentes dimensões afetivas. Com um vasto leque de possibilidades, Scopelliti e Giuliani (2004) apontam o que pode contribuir para que as pessoas se sintam restauradas durante seu momento de folga. Afirmam, também, que a interação social e as relações com os amigos em experiências restauradoras podem ser mais importantes para os jovens do que para os adultos e idosos. Também apresentam a hipótese de que as relações com os membros da família desempenham um papel mais forte para os adultos e idosos do que para os jovens. Quanto à dimensão afetiva das experiências de restauração, a hipótese formulada é a de que o papel do entusiasmo desempenhado na restauração entre jovens difere do relaxamento, mais importante e relevante para os idosos e adultos. Essa hipótese também diz respeito ao tempo disponível para a restauração e o contexto em relação ao qual a necessidade de restauração emerge, podendo influenciar a caracterização das experiências em termos de relaxamento e excitação. $\mathrm{O}$ estudo também avalia a interação social e o tipo de atividade praticada que melhor possa caracterizar o momento de restauração (Berto, 2007; Scopelliti \& Giuliani, 2004).

Com Korpela e colaboradores (K. Korpela, 1989; K. Korpela, Hartig, Kaiser, \& Fuhrer, 2001; K. M. Korpela, 1992), surge a ideia do uso do conceito ambientes restauradores, ou local de restauração no sentido de autorregulação (self-regulation). Os autores sugerem que ir ao local favorito passa a ser o motivo de restauração e de mudança - sendo essa a característica desejada nesses locais, correspondendo a uma atividade autorreguladora.

Lugares restauradores, assim como lugares favoritos, são escolhidos de acordo com as experiências neles vividas. Isso também é mencionado no estudo de Ouellette et al. (2005), no qual se concluiu que a experiência prévia em um ambiente pode permitir uma melhor adequação entre os objetivos e as possibilidades disponíveis, fortalecendo o fator compatibilidade sugerido na ART.

Outra forma de pensar a possibilidade de estudos sobre ambientes restauradores refere-se aos momentos de lazer. Tradicionalmente, a literatura sobre restauração sublinhou a importância do relaxamento, enquanto o papel da emoção manteve-se largamente inexplorado. Em outras palavras, é provável que o lazer tenha um valor de restauração, pelo menos até certo ponto. Algumas pesquisas enfatizam diferentes possibilidades de restauração, classificando-as de forma a corresponder à disponibilidade de tempo para essa atividade. Embora seja evidente que nem toda situação de lazer é restauradora, nem toda experiência restauradora pode ser categorizada como lazer. Pals, Steg, Siero e van der Zee (2009) e Scopelliti e Giuliani (2004), sugerem que exista algum tipo de sobreposição entre lazer e restauração.

Com relação às diferentes preferências pessoais e à aparência do lugar, Galindo e Hidalgo (2005) demonstraram que os atributos estéticos não afetam a categorização de um local como atrativo ou pouco atrativo. É o fator restaurador do local que afeta a sua categorização como um local atrativo ou pouco atrativo (Purcell, Peron, \& Berto, 2001).

Outros estudos indicam que locais urbanos como locais históricos, culturais ou mesmo sacros podem ter qualidades de ambiente restaurador (Galindo \& Hidalgo, 2005; Kaplan, Bardwell, \& Slakter, 1993; Ouellette et al., 2005). Existem propostas que investigam os mais diferentes ambientes e/ou as atividades relacionadas a lojas, cassinos, atrações em um zoológico, loja com jogos eletrônicos (arcade) e cafés com as possibilidades de restauração (Finlay, Marmurek, Kanetkar, \& Londerville, 2007; Joye, Willems, Brengman, \& Wolf, 2010; Pals et al., 2009; Rosenbaum, 2009).

A proposta de Felsten (2011), buscou compreender se as escolhas por diferentes ambientes restauradores não estariam ligadas à teoria do traço de personalidade, uma vez que muitas pesquisas a respeito de ambientes restauradores passam pela 
dicotomia entre ambiente natural $\mathrm{X}$ ambiente social e/ou construído.

Uma proposta atribui à fluência perceptual (perceptual fluency) a razão pela qual alguns ambientes são rapidamente compreendidos pelo cérebro humano. Joye (Joye, 2007; Joye \& van den Berg, 2012) argumentou que o cérebro humano possui mecanismos que otimizam o processamento e a decodificação de informações que possuem características fractais. $\mathrm{O}$ autor enfatizou que ao ser exposto a elementos de características fractais, isto é, a elementos que, mesmo depois de divididos em partes, mantêm as semelhanças com os objetos originais, o cérebro percebe a fluência e interpreta de forma diferente, reduzindo a atenção e, consequentemente, o estresse. Para o autor, não apenas os ambientes naturais possuem essa característica, mas também os ambientes construídos. Sugere-se, no entanto, que a escala com que esses elementos mantêm a característica fractal é algo basicamente percebido no ambiente natural.

Em suma, podemos afirmar que grande parte dos estudos aqui apresentados foi realizada em países onde há mudanças nítidas das estações do ano (Skärbäck, 2007; van den Berg, Hartig, \& Staats, 2007), com uso diferenciado de áreas recreativas, como parques e trilhas. Já que "os fatores pessoais, sociais, históricos e culturais referentes à 'experiência do lugar' devem ser considerados para se propor recomendações para o design e planejamento de ambientes" (Alves \& Gulwadi, 2008, p. 362), novos estudos devem ser conduzidos em contextos diferentes.

\section{Conclusão}

Historiadores, geógrafos, psicólogos discorrem sobre o processo histórico de oposição entre natureza (nature) e cultura (nurture) e expressam que a sociedade (cultura), é um esquecimento da natureza (Moscovici, 1975). A chamada modernidade tardia, que se distingui pela crença na razão e no progresso, parece medir este último pelo consumo, pelo sucesso pessoal e profissional. A ciência ecológica postula, por sua vez, que a comunidade de seres vivos e o nicho geofísico compõem uma unidade global, o ecossistema. Como conciliar este aparente paradoxo - a interdependência global com as necessidades de pessoas expostas ao ciclo estimulação/recuperação e que tendem a se comportar de maneira independente e autocentrada? Os estudos empíricos sugerem que as atividades em ambientes naturais reduzem o estresse da vida diária, promovem a capacidade de recuperação ante os desgastes cotidianos e ajudam a estabelecer vínculos emocionais com o ambiente proximal e distal. Os estudos aqui apresentados, realizados em sua maioria em países do hemisfério norte, onde a natureza já foi dominada, devem ser adaptados às particularidades de países como o Brasil, no qual a natureza não é vista, por alguns, como aliada, mas como uma inimiga a ser dominada. A limitação deste trabalho se prende ao fato de não ter conseguido apontar as variáveis determinantes para manter e preservar ambientes saudáveis ou para identificar as variáveis relevantes para a restauração das capacidades cognitivas e afetivas de grupos culturais e etários diversificados. O caminho parece ser longo, mas devemos refletir como fazer para conviver em/com uma sociedade que estime a natureza, não apenas pela quimera de voltar ao paraíso perdido, mas para valorizá-la como mais um elemento restaurador na vida das pessoas.

\section{Referências}

Altman, I., \& Wohlwill, J. F. (Orgs.). (1983). Behavior and the natural environment (Vol. 6). Nova Iorque e Londres: Plenum.

Alves, S. M. (2011). Ambientes restauradores. In S. Cavalcante \& G. A. Elali (Orgs.), Temas básicos em psicologia ambiental (pp. 44-52). Rio de Janeiro: Editora Vozes.

Alves, S. M., \& Gulwadi, G. B. (2008). Interação humana com ambientes naturais: Uma revisão no periódico environment and behavior. In J. Q. Pinheiro \& H. Gunther (Orgs.), Métodos de pesquisa nos estudos pessoa-ambiente (pp. 343-368). São Paulo: Casa do Psicólogo.

Bagot, K. L. (2004). Perceived restorative components: A scale for children. Children, Youth and Environments, 14(1), 120-140.

Baum, A., Fleming, R., \& Singer, J. E. (1985). Understanding environmental stress: Strategies for conceptual and methodological integration. In A. Baum \& J. E. Singer (Orgs.), Advances in Environmental Psychology (Vol. 5, pp. 185-205). Hillsdale: Lawrence Erlbaum Associates.

Berto, R. (2007). Assessing the restorative value of the environment: a study on the elderly in comparison with young adults and adolescents. International Journal of Psychology, 42(5), 331-341.

Bourassa, S. C. (1990). A paradigm for landscape aesthetics. Environment and Behavior, 22, 787-812.

Bringslimark, T., Hartig, T., \& Patil, G. G. (2009). The psychological benefits of indoor plants: A critical review of the experimental literature. Journal of Environmental Psychology, 29(4), 422-433. doi: 10.1016/j.jenvp.2009.05.001

de Kort, Y. A. W., \& Ijsselsteijn, W. A. (2006). Reality check: The role of realism in stress reduction using media technology. CyberPsychology \& Behavior, 9(2), 230-233. doi: 10.1089/cpb.2006.9.230

Evans, G. W., \& Cohen, S. (1987). Environmental stress. In D. Stokols \& I. Altman (Orgs.), Handbook of environmental psychology (Vol. 1, pp. 571610). Nova Iorque: Wiley.

Felsten, G. (2009). Where to take a study break on the college campus: An attention restoration theory perspective. Journal of Environmental Psychology, 29(1), 160-167. doi: 10.1016/j.jenvp.2008.11.006

Felsten, G. (2011, Setembro). Attention perceived stress recovery and restoration of natural attractive and less attractive, urban, and mixed scenes. Comunicação apresentada na 9th Biennial Conference on Environmental Psychology, Eindhoven, Netherlands. Resumo recuperado de http:// proceedings.envpsych2011.eu/files/doc/182.pdf

Finlay, K., Marmurek, H. H. C., Kanetkar, V., \& Londerville, J. (2007). Trait and state emotion congruence in simulated casinos: Effects on at-risk gambling intention and restoration. Journal of Environmental Psychology, 27(2), 166-175.

Galindo, M. P., \& Hidalgo, M. C. (2005). Aesthetic preferences and the attribution of meaning: Environmental categorization processes in the evaluation of urban scenes. International Journal of Psychology, 40(1), 19-26. doi: 10.1080/00207590444000104

Günther, H. (2011). Affordance. In S. Cavalcante \& G. A. Elali (Orgs.), Temas básicos em psicologia ambiental (pp. 21-27). Rio de Janeiro: Editora Vozes.

Han, K.-T. (2003). A reliable and valid self-rating measure of the restorative quality of natural environments. Landscape and Urban Planning, 64(4), 209-232. doi: 10.1016/S0169-2046(02)00241-4

Hartig, T., \& Staats, H. (2003). Guest editors' introduction: Restorative environments. Journal of Environmental Psychology, 23(2), 103-107. doi: 10.1016/S0272-4944(02)00108-1

Hartig, T., Evans, G. W., Jamner, L. D., Davis, D. S., \& Gärling, T. (2003). Tracking restoration in natural and urban field settings. Journal of 
Environmental Psychology, 23(2), 109-123.

Hartig, T., Kaiser, F. G., \& Bowler, P. A. (1997). Further development of a measure of perceived environmental restorativeness. Institute for Housing Research, 1-23. Recuperado de http://urn.kb.se/resolve?urn=urn:nbn:se:uu:diva-5762

Hartig, T., Korpela, K., Evans, G. W., \& Garling, T. (1997). A measure of restorative quality in environments. Scandinavian Housing and Planning Research, 14(4), 175-194.

Herzog, T. R., Black, A. M., Fountaine, K. A., \& Knotts, D. J. (1997). Reflection and attentional recovery as distinctive benefits of restorative environments. Journal of Environmental Psychology, 17(2), 165-170.

Herzog, T. R., Maguire, C. P., \& Nebel, M. B. (2003). Assessing the restorative components of environments. Journal of Environmental Psychology, 23(2), 159-170. doi: 10.1016/S0272-4944(02)00113-5

Holahan, C. J. (1986). Environmental psychology. Annual Review of Psychology, 37, 381-407. doi: 10.1146/annurev.ps.37.020186.002121

Joye, Y. (2007). Architectural lessons from environmental psychology: The case of biophilic architecture. Review of General Psychology, 11(4), 305-328.

Joye, Y., \& van den Berg, A. E. (2012). Restorative environments. In L. Steg, A. E., van den Berg \& J. I. M. De Groot (Orgs.), Environmental psychology: An introduction (pp. 57-66). West Sussex: BPS Blackwell.

Joye, Y., Willems, K., Brengman, M., \& Wolf, K. (2010). The effects of urban retail greenery on consumer experience: Reviewing the evidence from a restorative perspective. Urban Forestry and Urban Greening, 9(1), 57-64.

Kahn Jr, P. H., Friedman, B., Gill, B., Hagman, J., Severson, R. L., Freier, N. G., \& Stolyar, A. (2008). A plasma display window? The shifting baseline problem in a technologically mediated natural world. Journal of Environmental Psychology, 28(2), 192-199. doi: 10.1016/j.jenvp.2007.10.008

Kaplan, R. (1983). The role of nature in the urban context. In I. Altman \& J. F. Wohlwill (Orgs.), Behavior and the natural environment (Vol. 6, pp. 127159). Nova Iorque e Londres: Plenum.

Kaplan, R. (1984). Impact of urban nature: A theoretical analysis. Urban Ecology, 8(3), 189-197.

Kaplan, R., \& Kaplan, S. (1989). The experience of nature: a psychological perspective. Nova Iorque: Cambridge University.

Kaplan, R., Kaplan, S., \& Ryan, R. L. (1998). With people in mind: Design and management of everyday nature. Washington: Island.

Kaplan, S. (1987). Aesthetics, affect, and cognition. Environment and Behavior, 19(1), 3-32. doi: 10.1177/0013916587191001

Kaplan, S. (1995). The restorative benefits of nature: Toward an integrative framework. Journal of Environmental Psychology, 15(3), 169-182.

Kaplan, S., \& Kaplan, R. (Orgs.). (1982). Humanscape: Environments for people. Ann Arbor, MI: Ulrich's Books.

Kaplan, S., Bardwell, L. V., \& Slakter, D. B. (1993). The museum as a restorative environment. Environment and Behavior, 25(6), 725-742. doi: $10.1177 / 0013916593256004$

Kaplan, S., \& Talbot, J. F. (1983). Psychological benefits of a wilderness experience. Human Behavior \& Environment: Advances in Theory \& Research, 6, 163-203.

Karmanov, D., \& Hamel, R. (2008). Assessing the restorative potential of contemporary urban environment(s): Beyond the nature versus urban dichotomy. Landscape and Urban Planning, 86(2), 115-125. doi: 10.1016/j. landurbplan.2008.01.004

Korpela, K. (1989). Place-identity as a product of environmental self-regulation. Journal of Environmental Psychology, 9, 241.

Korpela, K., \& Hartig, T. (1996). Restorative qualities of favorite places. Journal of Environmental Psychology, 16(3), 221-233. doi: 10.1006/jevp.1996.0018

Korpela, K., Hartig, T., Kaiser, F. G., \& Fuhrer, U. (2001). Restorative experience and self-regulation in favorite places. Environment and Behavior, 33(4), 572-589. doi: 10.1177/00139160121973133

Korpela, K. M. (1992). Adolescents' favourite places and environmental selfregulation. Journal of Environmental Psychology, 12(3), 249-258.

Kuo, F. E., \& Sullivan, W. C. (2001). Aggression and violence in the inner city.
Environment and Behavior, 33(4), 543-571. doi: 10.1177/00139160121973124

Laumann, K., Gärling, T., \& Stormark, K. M. (2001). Rating scale measures of restorative components of environments. Journal of Environmental Psychology, 21(1), 31-44.

Mayer, F. S., Frantz, C. M., Bruehlman-Senecal, E., \& Dolliver, K. (2009). Why is nature beneficial? The role of connectedness to nature. Environment and Behavior, 41(5), 607-643.

Moscovici, S. (1975). Sociedade contra a Natureza (E. F. Alves, Trad.). Rio de Janeiro: Vozes.

Ouellette, P., Kaplan, R., \& Kaplan, S. (2005). The monastery as a restorative environment. Journal of Environmental Psychology, 25(2), 175-188.

Pals, R., Steg, L., Siero, F. W., \& van der Zee, K. I. (2009). Development of the PRCQ: A measure of perceived restorative characteristics of zoo attractions. Journal of Environmental Psychology, 29(4), 441-449. doi: 10.1016/j. jenvp.2009.08.005

Parsons, R., Tassinary, L. G., Ulrich, R. S., Hebl, M. R., \& Grossman-Alexander, M. (1998). The view from the road: Implication for the stress recovery and immunization. Journal of Environmental Psychology, 18(2), 113-139.

Perrin, J. L., \& Benassi, V. A. (2009). The connectedness to nature scale: A measure of emotional connection to nature? Journal of Environmental Psychology, 29(4), 434-440. doi: 10.1016/j.jenvp.2009.03.003

Purcell, T., Peron, E., \& Berto, R. (2001). Why do preferences differ between scene types? Environment and Behavior, 33(1), 93-106. doi: $10.1177 / 00139160121972882$

Regan, C. L., \& Horn, S. A. (2005). To nature or not to nature: Associations between environmental preferences, mood states and demographic factors. Journal of Environmental Psychology, 25(1), 57-66. doi: 10.1016/j. jenvp.2005.01.001

Rosenbaum, M. S. (2009). Restorative servicescapes: Restoring directed attention in third places. Journal of Service Management, 20(2), 173-191.

Scopelliti, M., \& Giuliani, M. V. (2004). Choosing restorative environments across the lifespan: A matter of place experience. Journal of Environmental Psychology, 24(4), 423-437. doi: 10.1016/j.jenvp.2004.11.002

Skärbäck, E. (2007). Landscape planning to promote well being: Studies and examples from Sweden. Environmental Practice, 9(3), 206-217.

Taylor, A. F., Kuo, F. E., \& Sullivan, W. C. (2002). Views of nature and selfdiscipline: Evidence from inner city children. Journal of Environmental Psychology, 22(1-2), 49-63.

Ulrich, R. S. (1983). Aesthetic and affective response to natural environment. In I. Altman \& J. F. Wohlwill (Orgs.), Behavior and the Natural Environment (Vol. 06, pp. 85-120). Nova Iorque: Plenum.

Ulrich, R. S. (1984). View through a window may influence recovery from surgery. Science, 224(4647), 420-421.

Ulrich, R. S., Simons, R. F., Losito, B. D., Fiorito, E., Miles, M. A., \& Zelson, M. (1991). Stress recovery during exposure to natural and urban environments. Journal of Environmental Psychology, 11(3), 201-230.

van den Berg, A. E. (2005). Health impacts of healing environments: A review of evidence for benefits of nature, daylight, fresh air, and quiet in healthcare settings. Groningen: The architecture of hospitals.

van den Berg, A. E., \& Custers, M. H. G. (2011). Gardening promotes neuroendocrine and affective restoration from stress. Journal of Health Psychology, 16(1), 3-11.

van den Berg, A. E., Hartig, T., \& Staats, H. (2007). Preference for nature in urbanized societies: Stress, restoration, and the pursuit of sustainability. Journal of Social Issues, 63(1), 79-96.

van den Berg, A. E., Koole, S. L., \& van der Wulp, N. Y. (2003). Environmental preference and restoration: (how) are they related? Journal of Environmental Psychology, 23(2), 135-146. doi: 10.1016/S0272-4944(02)00111-1

Wells, N. M. (2000). At home with nature: Effects of "greenness" on children's cognitive functioning. Environment and Behavior, 32(6), 775-795. doi: $10.1177 / 00139160021972793$ 
Sandra Christina Gressler, mestre em Environmental Design pela University of Missouri (MU), é doutoranda no programa em Psicologia Social, das Organizações e do Trabalho (PSTO) da Universidade de Brasília (UnB). Endereço para correspondência: Caixa Postal: 394, Dourados, MS - CEP: 79.804-970. E-mail: gressler.sandra90@gmail.com Isolda de Araújo Günther, PhD em Psicologia do Desenvolvimento pela Michigan State University (MSU), pós-doutora pela City University of New York e Carl von Ossietsy Universtiät, é pesquisadora Colaboradora Sênior no Instituto de Psicologia da Universidade de Brasília (UnB). E-mail: Isolda.gunther@gmail.com 